Audiology

Neurotology
Audiol Neurotol 2017;22:364-376

DOI: $10.1159 / 000488023$
Received: November 10, 2016

Accepted: February 26, 2018

Published online: June 28, 2018

\title{
Late Cochlear Implantation in Early-Deafened Adults: A Detailed Analysis of Auditory and Self-Perceived Benefits
}

\author{
Joke Debruyne ${ }^{a, b} \quad$ Miranda Janssen ${ }^{a, c} \quad$ Jan Brokx ${ }^{a, b}$ \\ a Department of Otorhinolaryngology, Head and Neck Surgery, Maastricht University Medical Center, Maastricht, The

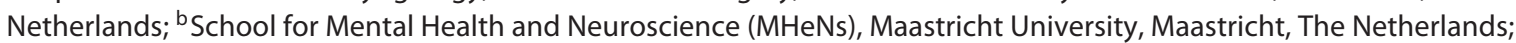 \\ 'Department of Methodology and Statistics, School for Public Health and Primary Care (CAPHRI), Maastricht University, \\ Maastricht, The Netherlands
}

\section{Keywords}

Cochlear implantation · Adult, early-deafened · Prelingual deafness - Speech perception - Subjective benefit .

Treatment outcome - Quality of life

\section{Abstract}

Objectives: It is known that early-deafened cochlear implant (Cl) users are a very heterogeneously performing group. To gain more insight into this population, this study investigated (1) postoperative changes in auditory performance over time based on various outcome measures, focusing on poor performers, (2) self-perceived outcomes, (3) relations between auditory and self-perceived outcomes, and (4) preimplantation factors predicting postoperative outcomes. Methods: Outcomes were assessed prospectively in a group of 27 early-deafened, late-implanted $\mathrm{Cl}$ users, up to 3 years after implantation. Outcome measures included open-set word and sentence recognition, closed-set word recognition, speech tracking and a questionnaire on self-perceived outcomes. Additionally, the relative influence of 8 preimplantation factors on $\mathrm{Cl}$ outcome was assessed with linear regression analyses. Results: Significant improvements were found for auditory performance measures and most of the questionnaire domains. Significant changes of the closed-

\begin{tabular}{ll}
\hline KARGER & $\begin{array}{l}\text { C } 2018 \text { The Author(s) } \\
\text { Published by S. Karger AG, Basel }\end{array}$ \\
E-Mail karger@karger.com & This article is licensed under the Creative Commons Attribution- \\
www.karger.com/aud & $\begin{array}{l}\text { NonCommercial-NoDerivatives 4.0 International License (CC BY- } \\
\text { NC-ND) (http://www.karger.com/Services/OpenAccessLicense). } \\
\text { Usage and distribution for commercial purposes as well as any dis- } \\
\text { tribution of modified material requires written permission. }\end{array}$
\end{tabular}

set word test, speech tracking and questionnaire were also found for a subgroup of poor performers. Correlations between auditory and self-perceived outcomes were weak and nonsignificant. Preoperative word recognition and preoperative hearing thresholds, both for the implanted ear, were significant predictors of postoperative outcome in the multivariable regression model, explaining $63.5 \%$ of the variation. Conclusions: Outcome measurement in this population should be adjusted to the patients' individual performance level and include self-perceived benefit. There is still a need for more knowledge regarding predictors of $\mathrm{Cl}$ outcomes in this group, but the current study suggests the importance of the preoperative performance of the ear to be implanted.

(c) 2018 The Author(s)
Published by S. Karger AG, Basel

\section{Introduction}

In the early years of cochlear implantation, adults with an early onset of deafness, i.e. before (prelingual) or during (perilingual) language development, displayed very limited benefits in speech-understanding tests [Skinner et al., 1992; Snik et al., 1997; Waltzman et al., 1992]. Consequently, they were often considered poor cochlear implant (CI) candidates. At the same time, studies reported 
that, in spite of these poor outcomes, satisfaction rates were high [Hinderink et al., 1995; Kaplan et al., 2003; Peasgood et al., 2003; Zwolan et al., 1996]. As speechcoding strategies evolved towards SPEAK, CIS and ACE from the late 90s onwards, studies showed that more beneficial results were possible for prelingually deafened adults [Schramm et al., 2002; Waltzman and Cohen, 1999; Waltzman et al., 2002]. A number of recent studies have demonstrated significant postoperative improvement in the mean scores of this group of CI users in various speech-understanding tests [Bosco et al., 2013; Caposecco et al., 2012; Klop et al., 2007; Rousset et al., 2016; Santarelli et al., 2008; Shpak et al., 2009; van Dijkhuizen et al., 2011; Yang et al., 2011]. Nevertheless, there are still large interindividual differences, with part of the early-deafened population obtaining scores slightly above or at $0 \%$ when speech understanding is assessed using open-set words or sentences. In this study, we argue that this does not necessarily mean that these CI users do not gain any auditory benefit from their CI, but rather that the tests used to evaluate performance might not be sensitive enough to detect more modest contributions of the implant to speech perception. In 2012, Caposecco et al. [2012] showed that while 18 of 38 CI users with earlyonset hearing loss were unable to attain more than $30 \%$ open-set speech discrimination postoperatively, they were all able to at least discriminate suprasegmental cues with their CI. Two other studies showed that poorly performing, prelingually deafened CI users were able to integrate auditory information from the CI with visual speech information, obtaining higher scores in the audiovisual condition [Craddock et al., 2016; Moody-Antonio et al., 2005]. These studies demonstrate the importance of including the ability to use suprasegmental and audiovisual cues when evaluating the benefit of cochlear implantation in this patient group. In this study, we wanted to investigate this further, by means of examining the added value of 2 nontraditional outcome measures to capture small, but relevant auditory benefits. Although recent studies have shown significant advancements in mean speech understanding scores for early-deafened CI users, it is still unclear when these subjects reach their maximum or so-called performance plateau. Postlingual CI users do generally not show further improvement after 1 year or even 6 months of CI use [Lenarz et al., 2012]. However, some studies assessing early-deafened CI users over time found that auditory performance continued to improve beyond 6 months or even 1 year after implantation [Santarelli et al., 2008; Shpak et al., 2009; Zeitler et al., 2012], whereas other studies observed no further increas-

Late Cochlear Implantation in Early-Deafened Adults es beyond the 1-year evaluation moment [van Dijkhuizen et al., 2016; Waltzman et al., 2002] or even earlier [Teoh et al., 2004]. This knowledge is important in order to guide patient expectations, and it may also influence how to design postimplantation rehabilitation. In addition to the evaluation of 2 nontraditional outcome measures, the current study therefore compares outcomes at regular intervals, up to 3 years after implantation.

There is increasing agreement that the impact of cochlear implantation should not only be evaluated with respect to changes in speech understanding performance, but also with respect to self-perceived changes in quality of life. High user satisfaction rates have consistently been reported in studies on early-deafened CI users, even in subjects with almost negligible gain in auditory performance [Bosco et al., 2013; Caposecco et al., 2012; Hinderink et al., 1995; Kaplan et al., 2003; Peasgood et al., 2003; Zwolan et al., 1996]. A number of studies have evaluated quality of life before and after implantation and found significant postoperative improvements, mainly in hearing-related quality of life questionnaires [Klop et al., 2007; Most et al., 2010; Schramm et al., 2002; Straatman et al., 2014; van Dijkhuizen et al., 2011], but also in hearingrelated domains of general health status questionnaires [Klop et al., 2007; Straatman et al., 2014]. Questionnaires like the Nijmegen Cochlear Implant Questionnaire (NCIQ) [Hinderink et al., 2000] are able to detect postoperative improvements for early-deafened CI users. Nevertheless it must be kept in mind that such questionnaires were developed to evaluate the benefit for postlingually deafened CI users and may therefore be less appropriate for early-deafened CI users with respect to, for example, the wording of questions (e.g., complexity) or the situations that are addressed (e.g., telephone use). In this study, the questionnaire applied is designed specifically to evaluate self-perceived benefit in early-deafened CI users. Moreover, the possible relation between self-perceived benefit and auditory benefit will be investigated, since they are both relevant in defining and measuring "successful implantation." It is pertinent to ask whether both outcome types provide the same information since this might affect patient counseling and possibly implant indications. Studies investigating this specific relation are scarce, however. In 2011, van Dijkhuizen et al. only found a significant correlation between speech perception outcomes and the subdomain "advanced sound perception" of the NCIQ, whereas both Peasgood et al. [2003] and Straatman et al. [2014] found no significant correlations between auditory outcome measures and scores on the Glasgow Benefit Inventory. Additionally, Straatman et al.

Audiol Neurotol 2017;22:364-376

DOI: $10.1159 / 000488023$ 
[2014] found no significant correlations between phoneme benefit scores and the generic Health Utilities Index 3 [Feeny et al., 2002] or the postoperative changes on the NCIQ. The authors hypothesized that prelingually deafened adults, in contrast to postlingually deafened adults, might be satisfied with just minimal improvements in hearing abilities.

So far we discuss here the significance of optimally measuring the outcome of cochlear implantation in this specific patient group. Another objective of this study is directed toward predicting preoperatively which patients will become good and/or satisfied CI users. Relevant patient characteristics can vary in many ways: the communication mode in which the patient has been raised, whether the hearing loss had a pre- or perilingual onset, the amount of residual hearing, whether or not hearing aids have been used, etc. It would be very useful to know which of these preimplantation factors are related to postoperative outcomes. The results of studies performing correlation analysis or multiple regression analysis in groups of prelingual CI users have been ambiguous. Study samples are often small, and a wide range of patient factors are considered that are not uniformly defined across studies. Although not conclusive over all studies, significant relations have been found between CI outcome and a patient's own speech intelligibility [van Dijkhuizen et al., 2016], communication mode [Caposecco et al., 2012; Rousset et al., 2016; Yang et al., 2011; Zeitler et al., 2012], and preoperative speech-understanding scores [Caposecco et al., 2012; Kraaijenga et al., 2016; Rousset et al., 2016; van der Marel et al., 2015; Yang et al., 2011]. At the same time, duration of deafness [Caposecco et al., 2012; Kraaijenga et al., 2016; van der Marel et al., 2015; van Dijkhuizen et al., 2016; Yang et al., 2011] and etiology [Kraaijenga et al., 2016; Zeitler et al., 2012] do not seem to be related to CI outcomes in this patient group.

In summary, the current study had 4 objectives. The first was to examine in detail postoperative changes in auditory performance in a group of late-implanted, earlydeafened adult CI users. For this purpose, both standard open-set word and sentence recognition tests were used pre- and postoperatively, as well as 2 less commonly used tests, hereafter referred to as "nontraditional" tests. The first of these 2 tests is speech tracking (ST) [De Filippo and Scott, 1978], which evaluates changes in the best-aided, audiovisual communicative abilities. The second is the monosyllable-trochee-spondee (MTS) test [Erber and Alencewicz, 1976], which assesses changes in closed-set (suprasegmental) word recognition. It was hypothesized that these nontraditional tests could demonstrate clear postoperative changes in subjects who show no or only limited improvements in the standard open-set auditory tests. We also wanted to determine if and when auditory performance reaches a plateau in this patient population. Therefore measurements were done up to 3 years after implantation. A second objective was to implement and evaluate a questionnaire that would be sensitive to the specific, self-perceived advancements of early-deafened CI users. The third study objective was to evaluate the relation between changes in auditory performance and changes in the subjective evaluation questionnaire. The final and fourth aim was to identify which preimplantation factors are related to postoperative speech-understanding scores with the CI. The investigated preimplantation factors were: preoperative pure-tone average (PTA) hearing loss in the better ear, preoperative PTA in the implanted ear, preoperative best-aided word recognition score, preoperative word recognition score in the implanted ear, communication mode at implantation, hearing aid use at implantation, preoperative hearing aid use in the implanted ear and age at onset of deafness (pre- vs. perilingual).

\section{Materials and Methods}

\section{Participants}

This article presents prospective data, gathered since 2010, on 27 adult early-deafened CI users who received an implant at the Maastricht University Medical Center (MUMC+). The Medical Ethical Committee of the MUMC+ deemed that the study fulfilled ethical requirements since it was an extension of regular patient care. All subjects who met the following criteria were included in the study: unilateral implantation, acquisition of deafness or severe hearing impairment at or before the age of 4 years and implantation in adulthood ( $>18$ years). In order to receive a CI at the MUMC+, early-deafened subjects additionally needed to be sufficiently motivated and receptive to auditory communication. There were no further exclusion criteria. Only subjects who had at least 1 year experience with the CI were included.

Twenty-seven subjects met the inclusion criteria. The mean age at implantation was 45 years (range: $20-71$ years). A prelingual onset of deafness or severe hearing loss, defined as an age at onset of $\leq 12$ months, was found in 22 or $81 \%$ of subjects; the remaining $5(19 \%)$ had a perilingual onset ( $>12$ but $<48$ months). Nineteen subjects $(70 \%)$ were oral communicators, the remaining $8(30 \%)$ used a combination of oral and manual communication. All but 4 subjects (85\%) were using at least 1 hearing aid at the time of the implantation, and 18 subjects (67\%) were wearing a hearing aid in the ear that was subsequently implanted. An overview of relevant subject characteristics and a number of summary statistics can be found in Table 1. All subjects had followed the extensive rehabilitation program which is the standard postimplantation care at the MUMC+, and all but 1 were regular users of their device. The latter subject, S15, became a nonuser after 1 year of CI use because she could not become accustomed to the sound of the implant. The 


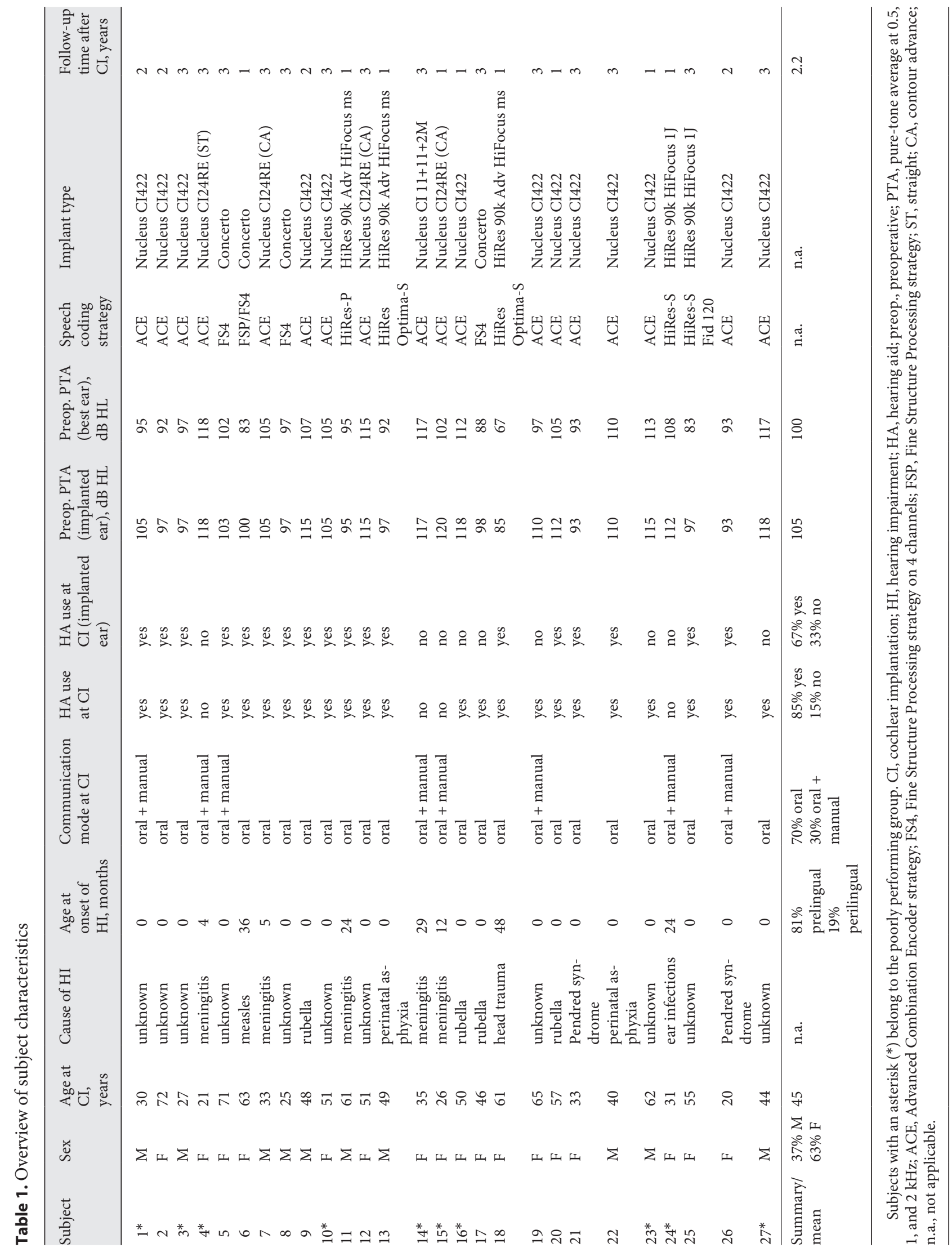


postimplantation follow-up time was 3 years for 14 subjects, 2 years for 4 subjects, and 1 year for the remaining 9 subjects (mean 2.2 years). Complications occurred neither during CI surgery nor during the postoperative rehabilitation period.

\section{Outcome Measures}

The preoperative PTA hearing loss at $0.5,1$, and $2 \mathrm{kHz}$ was calculated for each subject, both for the implanted ear and for the best ear (Table 1). Open-set word recognition was evaluated in free field at 65 and $75 \mathrm{~dB}$ SPL by means of the phoneme score of the Dutch, monosyllabic, consonant-nucleus-consonant (CNC) word test [Bosman and Smoorenburg, 1995]. The maximum percentage correct was gathered in the best-aided auditory-only condition (with 1 or 2 hearing aids preoperatively; postoperatively in the condition used in daily life: either CI alone or CI + contralateral hearing aid) and additionally in the CI-alone condition. The second open-set test was a Dutch sentence recognition test, with a variable volume setting according to the subjects' preference, administered in the best-aided, auditory-only condition. The result is expressed as a syllable score. The third test, ST, is a structured, "conversation-like" task that combines sentence identification with auditory comprehension [De Filippo and Scott, 1978]. The amount of time needed to repeat a text, which is read live and out loud to the subject, determines the score, expressed as the number of words per minute (wpm). The ST test was administered in the best-aided, auditory-visual condition. The texts used were specifically designed for early-deafened, Dutch CI users [Boons and Debruyne, 2011]. In poor performers, the MTS test [Erber and Alencewicz, 1976] was used as a test for closed-set (suprasegmental) word recognition. This 12 -item test assesses both word identification (word score) as well as identification of suprasegmental cues (suprasegmental score). Administration of the MTS test was at $65 \mathrm{~dB}$ SPL in the best-aided, auditory-only condition. If no hearing aids were worn in the preoperative condition of the open-set word, sentence or MTS test, or if the test was not administrable, a score of $0 \%$ was assigned.

A questionnaire to assess subjective outcomes was constructed specifically for early-deafened CI users. The questionnaire consists of 5 subscales: primary sound processing, sense of safety, ease of communication, social aspects of hearing, and self-confidence. Online suppl. Appendix A (for all online suppl. material see www. karger.com/doi/10.1159/000488023) contains information regarding the development and subsequent reliability analyses. The translation of the questionnaire is presented in online suppl. Appendix B.

Auditory tests were administered preoperatively, 6 months, and 1 year postoperatively. For some subjects they were also administered at 2 and 3 years postoperatively, according to available follow-up time. Subjects also completed the questionnaire dedicated to early-deafened CI users at each of these evaluation moments, except at 6 months postoperatively.

\section{Data Analysis}

To evaluate the postoperative changes in auditory performance up to 3 years after implantation (first study objective), with decreasing subject numbers over time, multilevel linear regression modeling was performed. The applied covariance structure chosen was based on likelihood ratio testing and the bayesian information criterion. Auditory performance changes over a specific time period were then evaluated with pairwise comparisons. Bonferroni-
Holm adjustments of the standard $\alpha$-value of 0.05 were made in case of multiple comparisons. If the sample size was small, which was the case when considering a subgroup of subjects, the nonparametric Wilcoxon signed-rank test was applied to pairwise comparisons. Descriptive statistics of the difference or change scores between preoperative and 1-year postoperative measurements were also obtained.

For the questionnaire (second study objective), the number of subjects with available data decreased with follow-up time. Missing responses on questionnaire items were replaced if, for a patient, the amount of missing values was $\leq 50 \%$ for a subscale. In case of $>50 \%$ missing values, the subject was excluded from further analysis of that particular subscale. Multiple imputation was used to generate 40 data sets. These data sets were then analyzed separately, and finally a single (pooled) multiple imputation estimate and its standard error were calculated by combining the estimates and standard errors obtained from each completed data set using " $\mathrm{Ru}$ bin's rules" [Little and Rubin, 2002; Rubin, 1987]. The imputation method was determined by the "automatic" method in SPSS multiple imputation. Multilevel linear regression analyses with a compound symmetry covariance structure were performed to assess the course of the subscale sum scores and total sum score of the questionnaire over the different time periods. In case of multiple comparisons, $p$ values were again Bonferroni-Holm corrected, with $\alpha \leq 0.05$ being considered statistically significant.

To evaluate the relation between results of the questionnaire and speech performance tests (third study aim), the Spearman correlation coefficients were calculated given the small sample size and since some variables did not display a normal distribution. Correlations were calculated between the difference scores ( 1 year post- vs. preoperative) of both the CNC word test and the ST test on one side and the changes in outcome 1 year after implantation of the total score and subscale scores of the questionnaire on the other.

For the fourth study objective, the bivariate relations between preimplantation factors and the 1-year postoperative CNC word recognition score with CI were first explored with simple linear regression. Multivariable regression analysis, using a backward method, was then performed with those variables that were significant in the simple linear regression analysis. During the backward regression analysis, nonsignificant preimplantation factors were sequentially removed (removal criterion: $p<0.10$ ). In the final model there were then no more variables that met the removal criterion. The final model is presented with estimated coefficients, along with the "adjusted" $R^{2}$, which is how much of the total variation in word recognition 1 year postoperatively can be explained by the model, adjusted for the number of variables included. All analyses were performed using SPSS 22.0.

\section{Results}

\section{Study Objective 1: Changes in Auditory Performance}

Figure 1a, b, and c shows the individual and mean observed scores of the 27 subjects in the word recognition, sentence recognition and ST tests, respectively. Multilevel analysis showed a significant effect of the time of measurement for word recognition $(\mathrm{F}(4,19.41)=5.139, p=$ 
0.005), sentence recognition $(\mathrm{F}(4,21.89)=4.525, p=$ $0.008)$, and the ST test $(\mathrm{F}(4,78.31)=12.076, p<0.0001)$. After Bonferroni-Holm correction, pairwise comparisons revealed significantly higher scores for all postoperative measurements when compared to the preoperative situation, and this in all 3 tests, with the exception of the 2-year postoperative measurement of the sentence recognition test ( $p=0.07$ after correction). In the ST test, significant improvements were found 2 and 3 years postoperatively compared to the 1-year postoperative situation. No further differences were found between the postoperative measurements in any of the tests.

Although postoperative improvements were found for the entire subject group, the scores of a number of subjects remained very low, for some even at $0 \%$. Table 2 shows the preoperative and 1 -year postoperative scores of the individual subjects for the different outcome measures. The changes in score from preoperative to 1-year postoperative were added as difference scores. Subjects whose scores remained below $30 \%$ in the open-set word recognition test are labeled "poor performers" and marked with an asterisk in both Tables 1 and 2. The 30\% criterion applied was the same as that by Arisi et al. [2010] and Caposecco et al. [2012]. If the difference score of an individual subject in Table 2 was $\leq 5 \% / \leq 5 \mathrm{wpm}$, the result was considered to be stable (no change). Inspection of individual scores reveals that only 5 subjects (S7, S12, S13, S18, and S22) show an improvement for all 3 tests, i.e., open-set word recognition, sentence recognition, and ST. Seven more subjects (S2, S5, S6, S8, S9, S25, and S26) show an improvement in 2 of the 3 tests and have a stable score on the third. Subject 21 showed improvement in 2 tests, but there was no ST score available. None of these in total 13 subjects belong to the group of earlier defined "poor performers." The remaining subjects who neither show improvements in at least 2 out of 3 tests nor belong to the poor performers are S11, S17, S19, and S20. The performance of both S11 and S19 is more or less the same before and after cochlear implantation. S11 performs well, both preoperatively with 2 hearing aids, as well as 1 year postoperatively with hearing aid and CI. S19 displays a small improvement of postoperative word recognition, while scores for sentence recognition and ST remain unchanged. For S17 and S20 we see a discrepancy in their results, with improvements in 2 of the 3 tests, but declines on the third. When looking at the poor performers $(n=10)$, it can be seen that only S1 and S27 show an improvement of open-set word recognition, of 6 and $17 \%$, respectively. S10, on the other hand, performs $12 \%$ worse postoperatively. For the remaining poor per-

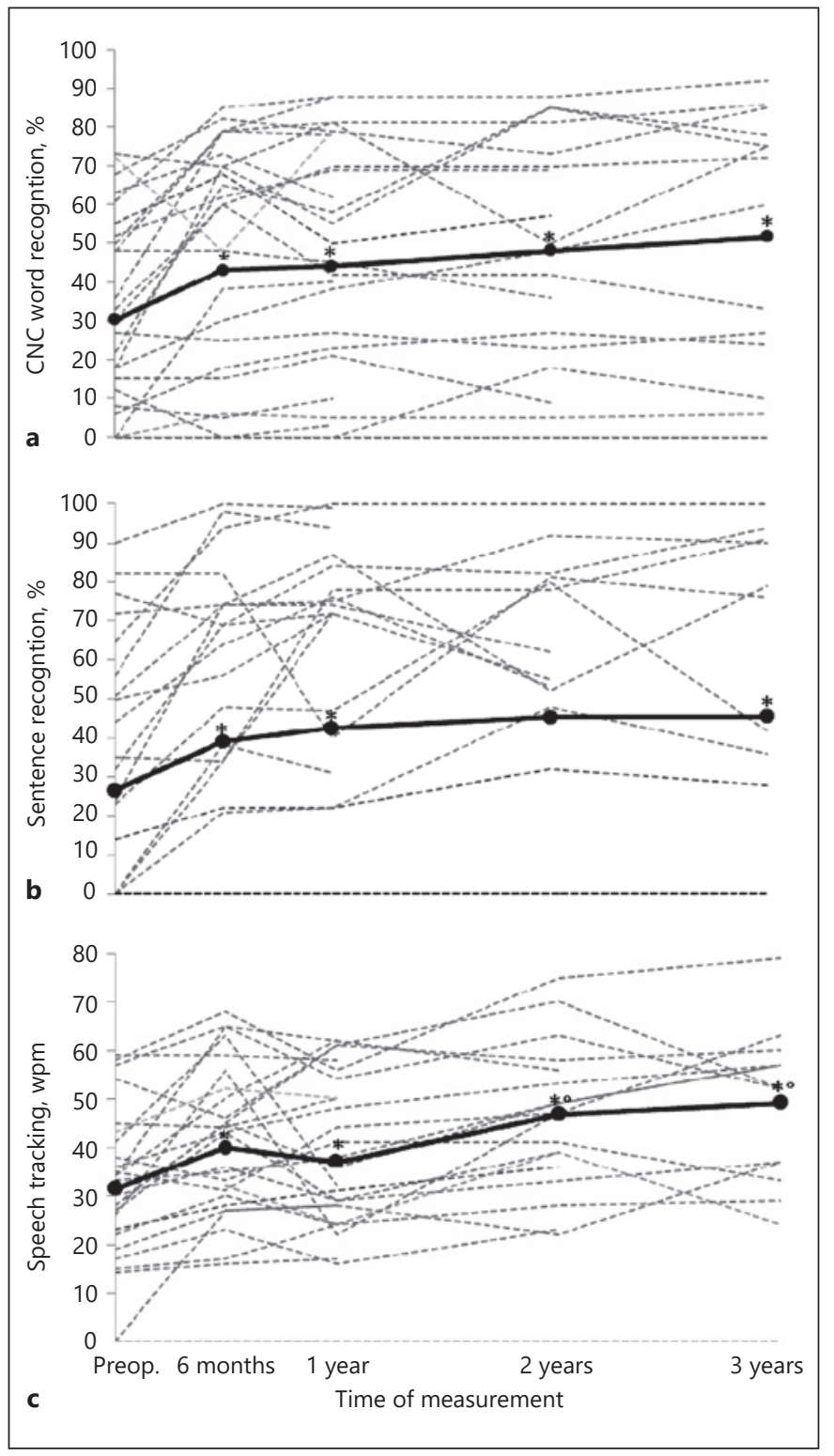

Fig. 1. Individual (striped lines) and mean (thick line) observed scores in the CNC word recognition (a), sentence recognition (b), and ST test (c) for $n=27$. Preoperatively, 6-month, 1-, 2-, and 3 -year postoperatively observed mean scores were 30.2, 43.2, 44.2, 48.1 , and $51.6 \%$ for the CNC word scores (a), respectively, 26.5, $38.9,42.4,45.3$, and $45.4 \%$ for the sentence recognition scores (b), and $31.4,39.9,37.0,46.8$, and 49 words per minute (wpm) for the ST test (c). For 3, 10, and 1 subject(s), respectively, data points remain at 0 in $\mathbf{a}, \mathbf{b}$, and $\mathbf{c}$. Significant differences after BonferroniHolm correction are indicated with an asterisk when compared to the preoperative situation and with a circle when compared to the 1-year postoperative situation.
Late Cochlear Implantation in

Early-Deafened Adults 
Table 2. Individual subject scores

\begin{tabular}{|c|c|c|c|c|c|c|c|c|c|c|c|c|c|c|c|}
\hline \multirow[t]{2}{*}{ Subject } & \multicolumn{3}{|c|}{$\begin{array}{l}\text { Open-set word recogni- } \\
\text { tion, } \%\end{array}$} & \multicolumn{3}{|c|}{ Sentence recognition, $\%$} & \multicolumn{3}{|c|}{$\begin{array}{l}\text { Speech tracking AV, } \\
\text { wpm }\end{array}$} & \multicolumn{3}{|c|}{ MTS word score, $\%$} & \multicolumn{3}{|c|}{$\begin{array}{l}\text { MTS suprasegmental } \\
\text { score, } \%\end{array}$} \\
\hline & preop. & $\begin{array}{l}1 \text { year } \\
\text { postop. }\end{array}$ & $\Delta$ score & preop. & $\begin{array}{l}1 \text { year } \\
\text { postop. }\end{array}$ & $\Delta$ score & preop. & $\begin{array}{l}1 \text { year } \\
\text { postop. }\end{array}$ & $\Delta$ score & preop. & $\begin{array}{l}1 \text { year } \\
\text { postop. }\end{array}$ & $\Delta$ score & preop. & $\begin{array}{l}1 \text { year } \\
\text { postop. }\end{array}$ & $\Delta$ score \\
\hline $1^{*}$ & 15 & 21 & 6 & 0 & 0 & 0 & 17 & 16 & -1 & 48 & 52 & 4 & 88 & 64 & -24 \\
\hline 2 & 55 & 50 & -5 & 50 & 72 & 22 & 23 & 31 & 8 & & & & & & \\
\hline 5 & 68 & 79 & 11 & 72 & 87 & 15 & 27 & 29 & 2 & & & & & & \\
\hline 6 & 48 & 88 & 40 & 56 & 94 & 38 & 45 & 50 & 5 & & & & & & \\
\hline 7 & 33 & 70 & 37 & 32 & 84 & 52 & 54 & 61 & 7 & & & & & & \\
\hline 8 & 61 & 88 & 27 & 65 & 100 & 35 & 58 & 56 & -2 & & & & & & \\
\hline 9 & 48 & 45 & -3 & 51 & 74 & 23 & 35 & 44 & 9 & & & & & & \\
\hline $10^{*}$ & 12 & 0 & -12 & 0 & 0 & 0 & 38 & 41 & 3 & 32 & 12 & -20 & 56 & 52 & -4 \\
\hline $15^{*}$ & 0 & 3 & 3 & 0 & 0 & 0 & 0 & 0 & 0 & 0 & 8 & 8 & 16 & 48 & 32 \\
\hline $16^{*}$ & 0 & 0 & 0 & 0 & 0 & 0 & 0 & 28 & 28 & 0 & 28 & 28 & 0 & 64 & 64 \\
\hline 17 & 73 & 55 & 18 & 82 & 40 & -42 & 36 & 48 & 12 & & & & & & \\
\hline 18 & 72 & 79 & 7 & 35 & 72 & 37 & 43 & 50 & 7 & & & & & & \\
\hline 19 & 30 & 42 & 12 & 0 & 0 & 0 & 33 & 38 & 5 & & & & & & \\
\hline 20 & 0 & 40 & 40 & 0 & 31 & 31 & 41 & 32 & -9 & & & & & & \\
\hline 21 & 36 & 81 & 45 & 24 & 75 & 51 & 57 & - & - & & & & & & \\
\hline 22 & 18 & 38 & 20 & 0 & 22 & 22 & 34 & 54 & 20 & & & & & & \\
\hline $23^{*}$ & 8 & 10 & 2 & 0 & 0 & 0 & 15 & 24 & 9 & 24 & 32 & 8 & 48 & 68 & 20 \\
\hline $24^{*}$ & 0 & 0 & 0 & 0 & 0 & 0 & 14 & 17 & 3 & 48 & 40 & -8 & 68 & 68 & 0 \\
\hline 25 & 22 & 58 & 36 & 23 & 47 & 24 & 26 & 22 & -4 & & & & & & \\
\hline 26 & 52 & 69 & 17 & 44 & 76 & 32 & 30 & 29 & -1 & & & & & & \\
\hline
\end{tabular}

Preoperative (preop.) and 1-year postoperative scores are presented, as well as the difference score between both evaluation moments. Mean scores are given for all subjects and for the subgroup of poor performers ( $n=10$, indicated with an asterisk behind the subject number). AV, auditory-visual condition; wpm, number of words per minute; MTS, monosyllable-trochee-spondee; $\Delta$, difference.

formers (S3, S4, S14, S15, S16, S23, and S24), phoneme scores in the open-set CNC word test remain stable. Only 1 of the poor performers (S3) improves more than $5 \%$ in the sentence recognition test and many obtain a $0 \%$ score, due to the test not being administrable. In contrast, scores of the ST and particularly the MTS test show a much greater variation, with improvements for some subjects (S3, S4, S14, S15, S16, S23, and S27) and stable results or even slight declines for others (S1, S10, and S24).
In Figure 2, as well as at the bottom of Table 2, the mean observed scores of the 10 poorly performing subjects are presented. S14 was excluded from the ST test because there was no preoperative measurement. A statistically significant improvement from a mean preoperative score of 38.4 to a mean postoperative score of $66.4 \%$ was found for the suprasegmental score of the MTS test $(p=0.038)$. For the word score of the MTS test, the improvement from a mean preoperative score of 20.8 to $35.2 \%$ postoperatively was nonsignificant $(p=0.092)$. In the ST test, a small but significant improvement from a 
Fig. 2. Preoperative and 1-year postoperative results of the poorly performing group. Error bars represent the standard error of the mean. Asterisks indicate a significant difference compared to the preoperative situation.

Fig. 3. Mean observed scores (expressed in percent) of the questionnaire for the $5 \mathrm{sep}$ arate subscales and the total questionnaire. Error bars represent the standard error of the mean. The number is $16-20$ for the preoperative and 1-year postoperative measurements, 13-14 for the 2-year postoperative measurement and 11 for the 3-year postoperative measurement. Asterisks indicate a significant difference after Bonferroni-Holm correction, compared to the preoperative situation.
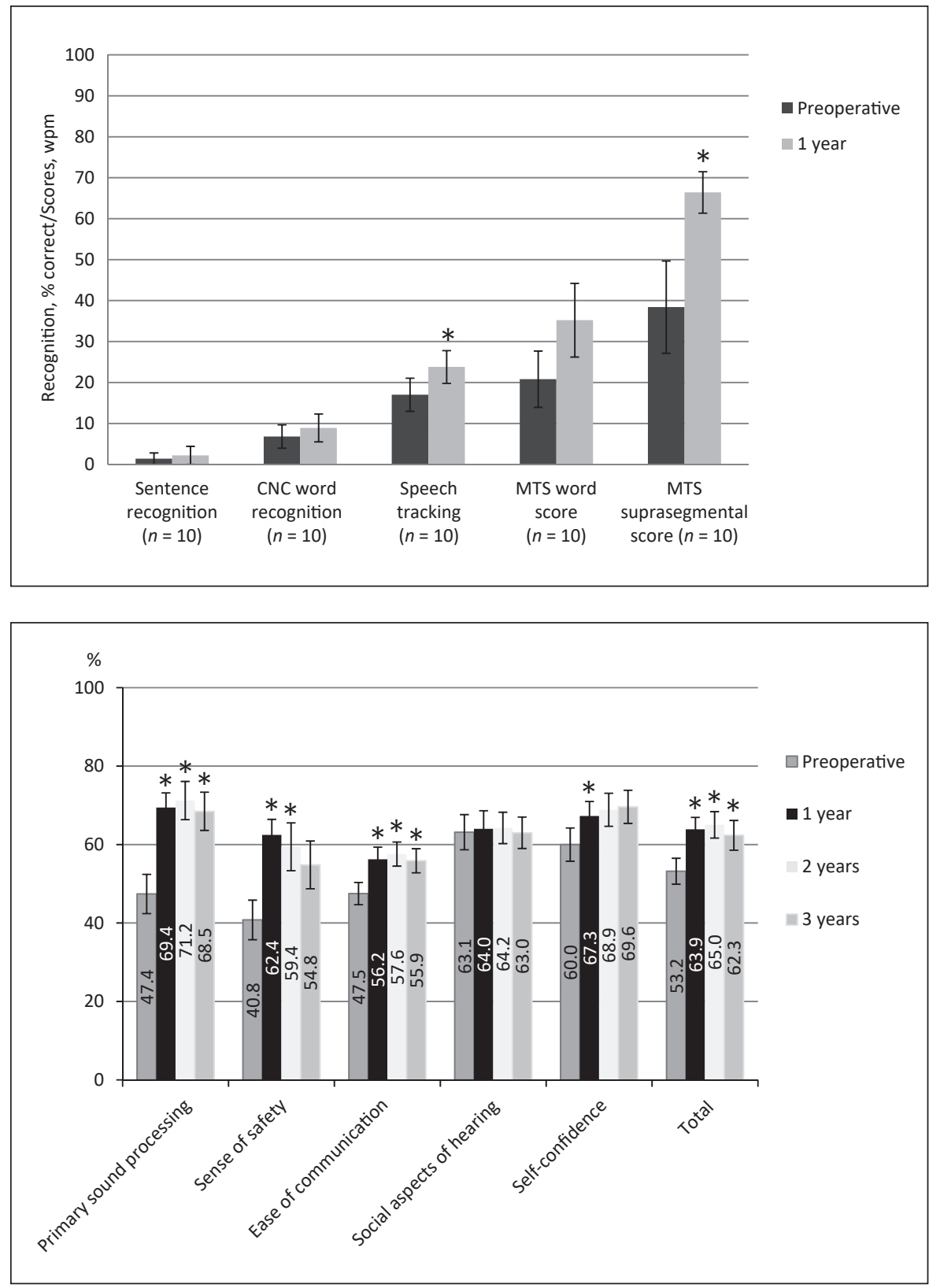

mean of $17 \mathrm{wpm}$ preoperatively to a mean of $23.8 \mathrm{wpm}$ postoperatively ( $p=0.017$ ) was seen. There was no improvement in the mean open-set word and sentence recognition scores after 1 year of CI use.

\section{Study Objective 2: Self-Perceived Benefits}

Questionnaire scale scores were obtained before and 1 year postoperatively for 16-20 subjects (depending on the subscale) and at 2 and 3 years postoperatively for $13-$ 14 and 11 subjects, respectively (Fig. 3). Multilevel analy- sis found significant improvements 1 year postoperatively compared to preoperative values for the total questionnaire score and each of the separate subscales except for "social aspects of hearing." The largest mean improvements were seen for primary sound processing (from $47.4 \%$ preoperatively to $69.4 \% 1$ year after) and sense of safety (from $40.8 \%$ preoperatively to $62.4 \% 1$ year after); the total score improved from $53.2 \%$ preoperatively to $63.9 \% 1$ year postoperatively. In the long term, scores remained significantly higher than preoperatively for the 
Table 3. Regression analyses

\begin{tabular}{|c|c|c|c|c|c|c|c|c|}
\hline \multirow[t]{2}{*}{ Preimplantation factors } & \multicolumn{4}{|c|}{ Simple regression } & \multicolumn{4}{|c|}{ Multivariable regression } \\
\hline & $\mathrm{B}$ & SE B & $\beta$ & $p$ & $\mathrm{~B}$ & SE B & $\beta$ & $p$ \\
\hline Preop. PTA (better ear) & -1.396 & 0.368 & -0.605 & 0.000 & ns & & & \\
\hline Preop. PTA (implanted ear) & -2.145 & 0.379 & -0.750 & 0.000 & -1.670 & 0.382 & -0.584 & 0.000 \\
\hline Preop. CNC word recognition (best-aided) & 0.759 & 0.162 & -0.685 & 0.000 & ns & & & \\
\hline Preop. CNC word recognition (implanted ear) & 0.828 & 0.205 & -0.629 & 0.000 & 0.472 & 0.176 & 0.359 & 0.013 \\
\hline Hearing aid use at implantation: yes/no & -38.957 & 13.416 & -0.502 & 0.004 & ns & & & \\
\hline Hearing aid use at implantation (implanted ear): yes/no & -37.278 & 9.006 & -0.638 & 0.000 & ns & & & \\
\hline Pre- vs. perilingual onset of deafness & 3.945 & 14.167 & 0.056 & 0.391 & & & & \\
\hline Communication mode at implantation: oral vs. oral + manual & -18.026 & 11.519 & -0.299 & 0.065 & & & & \\
\hline
\end{tabular}

Values for the unstandardized regression coefficient (B), standard error (SE) of B, standardized regression coefficient beta ( $\beta$ ), and the significance values $p$ are given for the regression of $\mathrm{CNC}$ word recognition score with CI only, 1 year after implantation, on 8 investigated preimplantation factors. For the simple regression the standardized coefficients $\beta$ represent the correlations between the preimplantation factors and the outcome variable. For the multivariable regression, 2 preimplantation factors remained as significant predictors in the final model after backward analysis. The adjusted $R^{2}$ for the final model was 0.635 . PTA, pure-tone average at 0.5 , 1 , and $2 \mathrm{kHz}$; ns, not significant. $n=27$.

total questionnaire and for the subscales "primary sound processing" and "ease of communication." This was not the case for "self-confidence" ( 2 and 3 years postoperatively) and "sense of safety" ( 3 years postoperatively). Total questionnaire scores were available for 5 of the 10 poorly performing subjects and showed a significant improvement of almost $11 \% 1$ year postoperatively $(\mathrm{z}=$ $-2.023, p=0.043)$.

\section{Study Objective 3: Relations between Auditory and Self-Perceived Benefits}

Correlation analyses of the changes in auditory performance with the changes in the questionnaire 1 year postoperatively revealed very weak to weak, nonsignificant correlations. Spearman correlations between the questionnaire (subscales and total questionnaire) and the difference scores of open-set word recognition are positive and range between 0.09 and $0.38(n=16-20)$; correlations between difference scores of the questionnaire and ST performance are close to zero (range: -0.08 and $0.12, n=14-20$ ).

\section{Study Objective 4: Predictive Value of Preimplantation}

Factors on Speech Recognition Scores

Simple regression analysis indicated that 6 of the 8 preimplantation factors were significant predictors for the 1-year postoperative CNC word recognition scores (left side of Table 3). Inclusion of these 6 factors as independent variables in a backward regression procedure resulted in a model including preoperative PTA $(p<0.0001)$ and preoperative $\mathrm{CNC}$ word recognition score $(p=$
0.013), both for the implanted ear, as predictors of the CNC word recognition score with CI 1 year postoperatively (right side of Table 3 ) with an explained variation of $63.5 \%$. The backward analyses performed are presented in online suppl. Appendix C.

\section{Discussion}

Study Objective 1: Changes in Auditory Performance

The first aim of this study was to evaluate the postoperative change in auditory performance of a group of early-deafened, late-implanted adult CI users by means of both traditional (open-set word and sentence recognition) and nontraditional (ST and the MTS test) tests, administered in the best-aided condition. A limitation concerning both the sentence recognition and ST test is that they are not validated. Results of the multilevel analysis showed a significant effect of the time of measurement for all 3 tests. Due to the relatively small number of subjects, which moreover decreased with follow-up time, these results do need to be interpreted with caution. Compared to the preoperative situation, significant improvements were present already after 6 months of CI use. The only exception was the 2-year postoperative measurement of the sentence recognition test, which lost its statistical significance after correction for multiple comparisons. Since no drop in performance was observed 2 years postoperatively (Fig. 1b), this lack of statistical significance might be explained by the reduction in statistical power due to 
a smaller sample size for that measurement. The fact that the change 3 years postoperatively once again attained statistical significance, even though the observed score remained the same with an even smaller sample size, is presumably due to the higher estimation of the mean, as predicted by the multilevel model. The mean word recognition scores of $44.1 \%$ (range $0-88 \%$ ) and mean sentence recognition scores of $42.4 \%$ (range $0-100 \%$ ) at the 1 -year postoperative measurement are comparable to those found in other studies, although it is unclear for some of these studies whether the reported scores were obtained with CI only or in the best-aided condition [Caposecco et al., 2012; Klop et al., 2007; Santarelli et al., 2008; van Dijkhuizen et al., 2011; Yang et al., 2011; Zeitler et al., 2012]. Consistently with the literature, we found extremely large differences between individual word and sentence recognition scores (Fig. 1a, b).

Until now, there has been no literature reporting ST for this patient group. Besides being administered in the best-aided condition, the ST test was performed with the aid of visual cues, bringing it more in line with everyday life communication. Although the absolute improvements were small for most subjects, the improvement was significant at the group level. Given that most of the subjects in this study were excellent lip readers, these apparently small changes are most likely explained by the fact that the added value of the CI becomes apparent in conjunction with the best communicative situation (e.g., lip reading). Results therefore show that it can be demonstrated that cochlear implantation can improve communication speed, when measured in a test situation that is as close to real-life communication as possible.

Another part of our first study objective was to see how performance in various tests evolved in the course of time. It is clear from Figure 1 that the largest changes occurred from before to 6 months after surgery, for all 3 tests. No significant improvements were found thereafter, except for 2- and 3-year postoperative versus 1-year postoperative time points in the ST test (Fig. 1c), although these may be attributable to the small decrease in performance 1 year postoperatively. Our results therefore conform to those on postlingual adults and do not support the hypothesis purported by Santarelli et al. [2008] that prelingually deafened CI users might show improved results in the long term due to remaining plasticity in the auditory cortical areas.

Descriptive analysis of the individual pre- and 1-year postoperative scores (Table 2) show that, of the 17 subjects that obtained more than $30 \%$ open-set word recognition
1 year after implantation, 13 showed a clear improvement in at least 2 out of the 3 tests including open-set word recognition, sentence recognition and ST. Two subjects (S11 and S19) scored the same before and after implantation, and 2 (S17 and S20) had mixed results, for which we have no explanation. On the other hand, the 10 subjects who scored below $30 \%$ word recognition 1 year postoperatively generally showed no improvement on open-set word or sentence recognition. One could draw the conclusion that there is no measurable auditory benefit of cochlear implantation in these subjects. However, when taking the results of the nontraditional outcome measures - ST and particularly MTS - into account, new information is added which allows us to distinguish between subjects that do show a measurable benefit with their CI and those who do not. Some subjects who are considered to be unsuccessful CI users do obtain auditory benefits. While no significant improvements were found for the open-set word and sentence recognition test, the suprasegmental score of the closed-set MTS test and the number of correctly repeated words per minute of the ST test did show significant improvements. Our hypothesis that nontraditional auditory measures are more suitable than traditional open-set, auditory-only speech tests to detect true auditory gains in poorly performing subjects is thus confirmed. It can therefore be advised to gear the choice of speech tests to the performance level of the subject, considering both standard and less traditional tests for an optimal monitoring of outcomes.

It is interesting to note that all 4 subjects who did not wear hearing aids preoperatively belong to the poorly performing group. These subjects did wear hearing aids during childhood but stopped wearing them as adolescents, presumably due to limited benefit. However, if we further compare the characteristics of those poorly performing subjects who show no postoperative improvement in any of the tests with those that do, there are no clear differences to be found in terms of communication mode, age at implantation, or hearing aid use.

\section{Study Objective 2: Self-Perceived Benefits}

Subjective experience after cochlear implantation should be an integral part of the evaluation protocol. A limitation of the questionnaire used in this study, which was specifically designed for the early-deafened group of CI users, is of course the lack of sufficient validation. Results on the questionnaire revealed that subjective scores were significantly higher after implantation for the subscales "primary sound processing," "sense of safety," and "ease of communication." Other studies that assessed hear- 
ing-related quality-of-life before and after implantation in this patient group also found significant postoperative improvements when using the NCIQ [Klop et al., 2007; Straatman et al., 2014; van Dijkhuizen et al., 2011], Performance Inventory for Profound and Severe Loss [Schramm et al., 2002], or a number of self-reporting questionnaires [Most et al., 2010]. In our study there was no effect of the $\mathrm{CI}$ on the subscale "social aspects of hearing," and the effect on "self-confidence" was only significant 1 year postoperatively. The subscale "social aspects of hearing" contains questions like "Do you like to go to parties or meetings?" and "How often do you feel left out by others?". A likely explanation for the absence of a significant change in this subscale may be the early onset of deafness. The subjects in this study had been participating in social situations with a severe hearing impairment for their entire lives, which is also illustrated by the relatively high preoperative scores in this subscale so that noticeable changes in this area are unlikely. The same reasoning may explain the slight changes found in the subscale "self-confidence." Other studies failed to find significant postoperative improvements for subscales related to self-confidence or self-esteem [Klop et al., 2007; Most et al., 2010], or found slightly smaller changes [Straatman et al., 2014]. Although it is hard to compare the content of the different questionnaires, it makes sense that the subscale "self-esteem" of the NCIQ also encompasses questions that are related to the subscale "social aspects of hearing" of the current study.

\section{Study Objective 3: Relations between Auditory and Self-Perceived Benefits}

Auditory gains on either CNC word recognition or ST did not appear to be related to benefit scores on the questionnaire. This might in part be attributable to the fact that there were significant improvements in the questionnaire scores for the subgroup of poor performers. Our results are in agreement with Straatman et al. [2014], although van Dijkhuizen et al. [2011] did find some significant correlations, but only with the subscale "advanced sound perception" of the NCIQ. This appears to indicate that in early-deafened CI users, quality of life benefit scores are generally independent of auditory gains. When judging whether an implantation is successful, both types of outcome measures should thus be taken into account, since they evaluate distinctly different aspects of cochlear implantation rehabilitation. A so-called poor performer might experience subjective benefits without improvement in open-set speech tests. Also, the fact that a correlation with the advancements in the ST test was not found, which - in contrast to the open-set word test - did show postoperative benefits even in the poorly performing group, suggests that self-perceived changes are not related to the improvements in day-today communication skills. It would be interesting to investigate further the relation between subjective outcomes and, for instance, the MTS test in a larger group of poor performers.

\section{Study Objective 4: Predictive Value of}

Preimplantation Factors on Speech Recognition Scores

Two of the 8 investigated preimplantation factors remained in the final multiple regression model: the preoperative $\mathrm{CNC}$ word recognition score and the preoperative PTA, both for the implanted ear, together explaining $63.5 \%$ of the variation in postoperative speech understanding with CI. This result needs to be interpreted with caution however, given that the regression analyses were based on data of only 27 subjects. Two recent studies also performed multiple regression analysis in groups of 58 and 43 early-deafened subjects, respectively [Kraaijenga et al., 2016; Rousset et al., 2016]. In the study by Kraaijenga et al. [2016], the preoperative best-aided word recognition score, and not the score of the ear to be implanted as in our study, was found to be the only significant predictor of the 1-year postoperative word recognition scores and explained $31 \%$ of the variation. In that same study, the preoperative PTA of the CI ear, which was the second significant factor in our model, correlated significantly with the outcome variable but lost its significance in the subsequent multivariable analysis. In the study by Rousset et al. [2016], the preoperative phoneme score of the ear to be implanted could, together with a standard score for receptive language abilities, account for $26.3 \%$ of the variation in postoperative speech perception scores. In their study however, the PTA of the implanted ear did not have a significant correlation with the outcome measure. Given that the word recognition score of the implanted ear was a significant predictor in both [Rousset et al., 2016] as in the current study, it seems worthwhile to include this factor in future studies.

While only 2 preimplantation factors remained as significant predictors in our final model, there were 4 more preimplantation factors correlating significantly with the outcome measure: preoperative best-aided CNC word recognition, preoperative PTA of the better ear, hearing aid use at implantation (yes/no) and hearing aid use at implantation in the implanted ear (yes/no). As mentioned previously, the preoperative best-aided word recognition score was a significant predictor in the multi- 
variable regression model of Kraaijenga et al. [2016]. Van der Marel et al. [2015] equally observed a significant correlation with preoperative phoneme scores. On the other hand, van Dijkhuizen et al. [2016] (after Bonferroni correction) and Zeitler et al. [2012] did not find a significant correlation with preoperative CNC scores. Similarly to the preoperative CNC scores, our study found the preoperative PTA of the implanted ear to be a significant predictor, whereas the significant correlation of the preoperative PTA of the better ear was dropped in the backward multiple regression modeling procedure. Both preimplantation factors regarding hearing aid use at implantation were also dropped in the backward procedure. In 2012, Caposecco et al. found that the time without an aid in the implant ear (at implantation) was a significant predictor, while van Dijkhuizen et al. [2016] found no significant correlation between postoperative speech perception and hearing aid use.

Finally, 2 of the 8 preimplantation factors did not show a significant correlation with our outcome measure: communication mode at implantation and pre- versus perilingual onset of deafness. The first is somewhat surprising, given that advantages for oral communicators are seen in most [Caposecco et al., 2012; Rousset et al., 2016; Yang et al., 2011; Zeitler et al., 2012], yet not all previous studies [van Dijkhuizen et al., 2016]. The factor regarding pre- versus perilingual onset of deafness has only been assessed by Caposecco et al. [2012]. In contrast to our results, this study did find a significant relation between the progressivity of the hearing loss and sentence recognition scores with CI.

\section{Conclusion}

This study showed that early-deafened but late implanted adult CI-users obtained significant auditory improvements, mainly within the first 6 months after implantation. It was found that, on average, auditory performance of poorly performing subjects increases but this can only be captured by using specific tests like ST or tests assessing more suprasegmental features of speech. On an individual subject level, these new outcome measures add valuable information and allow for more differentiation between poorly performing subjects. The current results emphasize the importance of using a different test battery for the auditory evaluation of this group of CI users.

An instrument assessing subjective experiences after CI should be an integral component of the evaluation protocol, given that an individually experienced benefit is not fully captured by the auditory tests.

Postoperative performance with CI could be explained to a large part by 2 preimplantation factors: preoperative PTA and preoperative CNC word recognition, both of the implanted ear. Studies in larger groups of early-deafened patients could contribute further to understanding the role that preimplantation factors have on CI outcome in this population.

\section{Disclosure Statement}

This work was supported by Cochlear Europe. The second author reviewed the manuscript and provided statistical support made possible by a grant from the Dutch Heinsius-Houbolt Foundation.

\section{References}

Arisi E, Forti S, Pagani D, Todini L, Torretta S, Ambrosetti U, Pignataro L: Cochlear implantation in adolescents with prelinguistic deafness. Otolaryngol Head Neck Surg 2010;142: 804-808.

Boons K, Debruyne J: Evaluatie van objectieve testmethodes voor prelinguaal dove volwassen CI-gebruikers. Logopedie 2011;24:48-52.

Bosco E, Nicastri M, Ballantyne D, Viccaro M, Ruoppolo G, Ionescu Maddalena A, Mancini $\mathrm{P}$ : Long term results in late implanted adolescent and adult CI recipients. Eur Arch Otorhinolaryngol 2013;270:2611-2620.

Bosman AJ, Smoorenburg GF: Intelligibility of Dutch CVC syllables and sentences for listeners with normal hearing and with three types of hearing impairment. Audiology 1995;34: 260-284.
Caposecco A, Hickson L, Pedley K: Cochlear implant outcomes in adults and adolescents with early-onset hearing loss. Ear Hear 2012;33: 209-220.

Craddock L, Cooper H, Riley A, Wright T: Cochlear implants for pre-lingually profoundly deaf adults. Cochlear Implants Int 2016; 17(suppl 1):26-30.

De Filippo CL, Scott BL: A method for training and evaluating the reception of ongoing speech. J Acoust Soc Am 1978;63:1186-1192.

Erber NP, Alencewicz CM: Audiologic evaluation of deaf children. J Speech Hear Disord 1976; 41:256-267.

Feeny D, Furlong W, Torrance GW, Goldsmith CH, Zhu Z, DePauw S, Denton M, Boyle M: Multiattribute and single-attribute utility functions for the health utilities index mark 3 system. Med Care 2002;40:113-128.
Hinderink JB, Krabbe PF, Van Den Broek P: Development and application of a health-related quality-of-life instrument for adults with cochlear implants: the Nijmegen Cochlear Implant Questionnaire. Otolaryngol Head Neck Surg 2000;123:756-765.

Hinderink JB, Mens LH, Brokx JP, van den Broek $P$ : Performance of prelingually and postlingually deaf patients using single-channel or multichannel cochlear implants. Laryngoscope 1995;105:618-622.

Kaplan DM, Shipp DB, Chen JM, Ng AH, Nedzelski JM: Early-deafened adult cochlear implant users: assessment of outcomes. J Otolaryngol 2003;32:245-249.

Klop WM, Briaire JJ, Stiggelbout AM, Frijns JH: Cochlear implant outcomes and quality of life in adults with prelingual deafness. Laryngoscope 2007;117:1982-1987.
Late Cochlear Implantation in

Early-Deafened Adults
Audiol Neurotol 2017;22:364-376

DOI: $10.1159 / 000488023$ 
Kraaijenga VJ, Smit AL, Stegeman I, Smilde JJ, van Zanten GA, Grolman W: Factors that influence outcomes in cochlear implantation in adults, based on patient-related characteristics - a retrospective study. Clin Otolaryngol 2016;41:585-592.

Lenarz M, Sonmez H, Joseph G, Buchner A, Lenarz T: Long-term performance of cochlear implants in postlingually deafened adults. Otolaryngol Head Neck Surg 2012;147:112-118.

Little RJA, Rubin DB: Statistical Analysis with Missing Data, ed 2. New York, Wiley, 2002.

Moody-Antonio S, Takayanagi S, Masuda A, Auer ET Jr, Fisher L, Bernstein LE: Improved speech perception in adult congenitally deafened cochlear implant recipients. Otol Neurotol 2005;26:649-654.

Most T, Shrem H, Duvdevani I: Cochlear implantation in late-implanted adults with prelingual deafness. Am J Otolaryngol 2010;31: 418-423.

Peasgood A, Brookes N, Graham J: Performance and benefit as outcome measures following cochlear implantation in non-traditional adult candidates: a pilot study. Cochlear Implants Int 2003;4:171-190.

Rousset A, Dowell R, Leigh J: Receptive language as a predictor of cochlear implant outcome for prelingually deaf adults. Int J Audiol 2016; 55(suppl 2):S24-S30.

Rubin DB: Multiple Imputation for Nonresponse in Surveys. New York, Wiley, 1987.

Santarelli R, De Filippi R, Genovese E, Arslan E: Cochlear implantation outcome in prelingually deafened young adults. A speech perception study. Audiol Neurootol 2008;13: 257-265.
Schramm D, Fitzpatrick E, Seguin C: Cochlear implantation for adolescents and adults with prelinguistic deafness. Otol Neurotol 2002; 23:698-703.

Shpak T, Koren L, Tzach N, Most T, Luntz M Perception of speech by prelingual pre-adolescent and adolescent cochlear implant users. Int J Audiol 2009;48:775-783.

Skinner MW, Binzer SM, Fears BT, Holden TA, Jenison VW, Nettles EJ: Study of the performance of four prelinguistically or perilinguistically deaf patients with a multi-electrode, intracochlear implant. Laryngoscope 1992; 102:797-806.

Snik AF, Makhdoum MJ, Vermeulen AM, Brokx JP, van den Broek P: The relation between age at the time of cochlear implantation and longterm speech perception abilities in congenitally deaf subjects. Int J Pediatr Otorhinolaryngol 1997;41:121-131.

Straatman LV, Huinck WJ, Langereis MC, Snik AF, Mulder JJ: Cochlear implantation in lateimplanted prelingually deafened adults: changes in quality of life. Otol Neurotol 2014; 35:253-259.

Teoh SW, Pisoni DB, Miyamoto RT: Cochlear implantation in adults with prelingual deafness. I. Clinical results. Laryngoscope 2004; 114:1536-1540.

Van der Marel KS, Briaire JJ, Verbist BM, Muurling TJ, Frijns JH: The influence of cochlear implant electrode position on performance. Audiol Neurootol 2015;20:202-211.
Van Dijkhuizen JN, Beers M, Boermans PP, Briaire JJ, Frijns JH: Speech intelligibility as a predictor of cochlear implant outcome in prelingually deafened adults. Ear Hear 2011;32: 445-458.

Van Dijkhuizen JN, Boermans PP, Briaire JJ, Frijns JH: Intelligibility of the patient's speech predicts the likelihood of cochlear implant success in prelingually deaf adults. Ear Hear 2016;37:e302-e310.

Waltzman SB, Cohen NL, Shapiro WH: Use of a multichannel cochlear implant in the congenitally and prelingually deaf population. Laryngoscope 1992;102:395-399.

Waltzman SB, Cohen NL: Implantation of patients with prelingual long-term deafness. Ann Otol Rhinol Laryngol Suppl 1999;177: 84-87.

Waltzman SB, Roland JT Jr, Cohen NL: Delayed implantation in congenitally deaf children and adults. Otol Neurotol 2002;23:333-340.

Yang WS, Moon IS, Kim HN, Lee WS, Lee SE, Choi JY: Delayed cochlear implantation in adults with prelingual severe-to-profound hearing loss. Otol Neurotol 2011;32:223-228.

Zeitler DM, Anwar A, Green JE, Babb JS, Friedmann DR, Roland JT Jr, Waltzman SB: Cochlear implantation in prelingually deafened adolescents. Arch Pediatr Adolesc Med 2012; 166:35-41.

Zwolan TA, Kileny PR, Telian SA: Self-report of cochlear implant use and satisfaction by prelingually deafened adults. Ear Hear 1996;17: 198-210. 